Romanistisches Jahrbuch

Begründet von

Olaf Deutschmann · Rudolf Grossmann · Hellmuth Petriconi · Hermann Tiemann 



\section{Romanistisches Jahrbuch}

Herausgegeben von

Andreas Dufter, Folke Gernert, Daniel Jacob, David Nelting, Christian Schmitt, Maria Selig, Susanne Zepp

Unter Mitwirkung von

Pedro M. Cátedra (Salamanca), Andreas Kablitz (Köln), Bernhard König (Köln), Joachim Küpper (Berlin), Jacques Neefs (Paris und Baltimore), Stephen Nichols (Baltimore), Marco Santagata (Pisa)

Redaktion

Rosemary Snelling-Gőgh und Yvonne Stork

\section{Band 72 2021}

\section{DE GRUYTER}


Hinweise zur Manuskripteinrichtung finden Sie auf der Website des Romanistischen Jahrbuchs: https://www.degruyter.com/journal/key/ROMA/html

ISSN 0080-3898

e-ISSN 1613-0413

Bibliografische Information der Deutschen Nationalbibliothek

Die Deutsche Nationalbibliothek verzeichnet diese Publikation in der Deutschen

Nationalbibliografie; detaillierte bibliografische Daten sind im Internet über http://dnb.dnb.de abrufbar.

(c) 2021 Walter de Gruyter GmbH, Berlin/Boston

Satz: jürgen ullrich typosatz, Nördlingen

Druck und Bindung: CPI books $\mathrm{GmbH}$, Leck

www.degruyter.com 


\section{Inhalt}

\section{Allgemein-Romanistischer Teil}

Chronik $2020-3$

\section{Aufsätze und Berichte -29}

Sarah Dessì Schmid (Tübingen)

Zur Beziehung von progressiven Verbalperiphrasen und states.

Ein erster Bericht aus Studien zu romanischen Sprachen - 31

Aurelia Merlan (München)

Rumänisch im deutschen Migrationskontext -63

Felix Tacke (Bonn)

Sprache, Genres und Diskurstraditionen. Kognitionslinguistische Modelle im Lichte der romanistischen Theoriebildung — 118

Stefan Feddern (Köln)

Boccaccios Überlegungen zum Ursprung der allegorischen Dichtung — 156

Yona Hanhart-Marmor (Jerusalem)

Like Father, Unlike Son: Reluctant Heirs in Contemporary Western

Literature - 194

Stephanie Wodianka (Rostock)

Stimmversagen, Sprachinfarkt und Zungenbremse? Posttridentinische Betrachtungsliteratur Italiens zwischen orazione mentale und orazione vocale - 218

Buchbesprechungen - Buchanzeigen - 247

Márton Gergely Horváth, Le français parlé informel. Stratégies de topicalisation (Barbara Wehr) — 249 
Franz Lebsanft/Felix Tacke (eds.), Manual of Standardization in the Romance Languages

(Laura Linzmeier) -268

Frédéric Nicolosi, Topic- und Focus-Markierung im Altitalienischen (Silvio Cruschina) -278

Andra Vasilescu/Mihaela-Viorica Constantinescu/Gabriela Stoica/Jonathan Russell White (eds.), Exploring Discourse Practices in Romanian (Daniel Biro) - 285

Peter-André Alt, „Jemand musste Josef K. verleumdet haben...“. Erste Sätze der Weltliteratur und was sie uns verraten

(Dietrich Scholler) - 293

Carmen Rivero, Humanismus, Utopie und Tragödie (Marília Jöhnk) - 298

Juana Christina von Stein, Melancholie als poetologische Allegorie.

Zu Baudelaire und Flaubert

(David Nelting) - 304

\section{Ibero-Romanistischer Teil}

\section{Aufsätze und Berichte - 309}

Juan Antonio Ennis (La Plata)/Claudio Soltmann (Santiago de Chile) Comunicación epistolar y colaboración científica entre especialistas alemanes en Sudamérica a fines del siglo XIX. Un estudio de la correspondencia entre Rodolfo Lenz y Robert Lehmann-Nitsche — 311

Sandra Schlumpf (Basel)

Spanisch in Afrika/Afrika in Spanien. Sprachliche Charakteristika von Spanischsprecherinnen und Spanischsprechern aus Äquatorialguinea in Madrid - 339

Juan-Carlos Conde (Salamanca)

Self-Fashioning and the Intersectional Self: Teresa de Cartagena by Teresa de Cartagena -388 
Jimena Gamba Corradine (Bern)

Itinerario de un texto de Erasmo: el Sermón de las misericordias de Dios en castellano $(1528,1544$ y 1549) -421

\section{Buchbesprechungen - Buchanzeigen - 447}

Teresa Hiergeist, Tiere der Arena - Arena der Tiere. Neuverhandlungen der Interspezies-Relationen in den aristokratischen Kampfspielen des siglo de oro (Frank Nagel) -449 
